\title{
Fingeriana dubia gen. nov. e sp. nov. de Cicadellini (Hemiptera, Auchenorrhyncha, Cicadellidae) do sudeste e sul do Brasil ${ }^{1}$
}

\author{
Rodney Ramiro Cavichioli ${ }^{2}$
}

1 Contribuição número 1394 do Departamento de Zoologia, Universidade Federal do Paraná.

2 Departamento de Zoologia, Universidade Federal do Paraná. Caixa Postal 19020, 81531-980 Curitiba, Paraná, Brasil. Bolsista do CNPq. E-mail: cavich@ufpr.br

\begin{abstract}
Fingeriana dubia gen. nov. and sp. nov. of Cicadellini (Hemiptera, Auchenorrhyncha, Cicadellidae) from Southeast and South of Brazil. New genus and new species of Cicadellini are proposed: Fingeriana dubia occurs at the citrus and coffee culture. The new genera is similar with Nielsonia Young, 1977 but, the shaft of the adeagus of the Fingeriana dubia is symmetrical with a basal unpaired process asymmetrical and plates narrowly triangular as long as pygofer.
\end{abstract}

KEY WORDS. Cicadellidae, Cicadellinae, Fingeriana gen. nov., Hemiptera, new species.

Young (1977) revisou a tribo Cicadellini, onde descreveu 91 gêneros novos e redescreveu outros 64 gêneros das regiões Neártica e Neotropical, exceto Cicadella Latreille, 1817 que é Paleártico e propondo 10 grupos de gêneros. Após esta revisão, foram poucos os gêneros descritos para as regiões Neártica e Neotropical. Hamilton (1985) descreveu Xyphon e o relaciou com Draeculacephala Ball,1901 tendo distribuição na região Neártica. MejDalani (1994) descreveu Neodayoungia para abrigar Tettigonia xanthonota Melichar, 1926, colocado em posição incerta por Young (1977), considerando-o próximo de Fonsecaiulus Young, 1977.

Nielson \& Godoy (1995) propuseram Gillonella relacionando-o com Nielsonia Young, 1977; Schistogonalia Young, 1977 e Macunolla Young, 1977, por apresentarem o edeago assimétrico. CAvichioli (1996), revalidou Alocha Melichar, 1926 e propôs Albiniana, ambos relacionados com Paromenia Melichar, 1926. CAviCHIOLI (1998) descreveu Clypelliana, o qual está relacionado com Trichogonia Young,1977. TAKIYA et al. (2001) propuseram Aurigoniella e relacionado-o com Syncharina Young, 1977 e Helocharina Melichar, 1926. CAvichioli (2001a, b) descreveu Wolfiniana e Jeepiulus, o primeiro relacionado com os gêneros do grupo Cicadella e o segundo com os gêneros Alocha Melichar, Albiniana Cavichiol e Paromenia Melichar. Todos esses gêneros, exceto Xyphon Hamilton, apresentam distribuição na região Neotropical.

O gênero e a espécie novos propostos neste trabalho, foram coletados em cultura de citros e café, nos estado de Minas Gerais, São Paulo e Paraná.

As informações contidas nas etiquetas foram transcritas entre aspas ("), sendo que, o conteúdo das linhas separadas por um ponto-e-vírgula (;).
A terminologia utilizada para os caracteres da morfologia é aquela de Young $(1968,1977)$, exceto para cabeça, onde se segue Hamilton (1981) e Mejadalani (1998) e para as estruturas da genitália das fêmeas, utiliza-se Nielson (1965). A metodologia para os estudos das genitálias dos machos e das fêmeas, seguiuse às técnicas descritas por OMAN (1949) e ZANOL (1988). As medidas são fornecidas em milímetros

\section{Fingeriana gen. nov.}

Espécie-tipo: Fingeriana dubia sp. nov.

Macho: 6,00; fêmea: 6,80.

Cabeça, em vista dorsal, projetada anteriormente, com comprimento mediano levemente mais do que metade da distância transocular e quase igual à distância interocular; margem anterior levemente angulada, sem carena entre a coroa e a face; ocelos localizados atrás da linha imaginária que tangencia os bordos anteriores dos olhos e equidistantes destes e da linha mediana; superfície da coroa lisa; lóbulos supra-antenais, em vista dorsal, pouco evidentes, em vista lateral, oblíquos e carenados dorsalmente. Fronte, mais longa do que sua maior largura, em vista lateral, intumescida, apresentando a porção mediana levemente achatada e com impressões musculares evidentes; sutura fronto-clipeal obscura medianamente; clípeo, basalmente mais largo, de perfil com contorno ao da fronte, levemente intumescido medianamente e apicalmente arredondado. Pronoto mais estreito do que a cabeça, de comprimento mediano igual 5/9 da distância entre os úmeros; margens laterais levemente convergentes anteriormente e a posterior com uma reentrância mediana; disco do pronoto com a superfície lisa, carena dorsopleural completa. Escutelo mais largo basalmente 

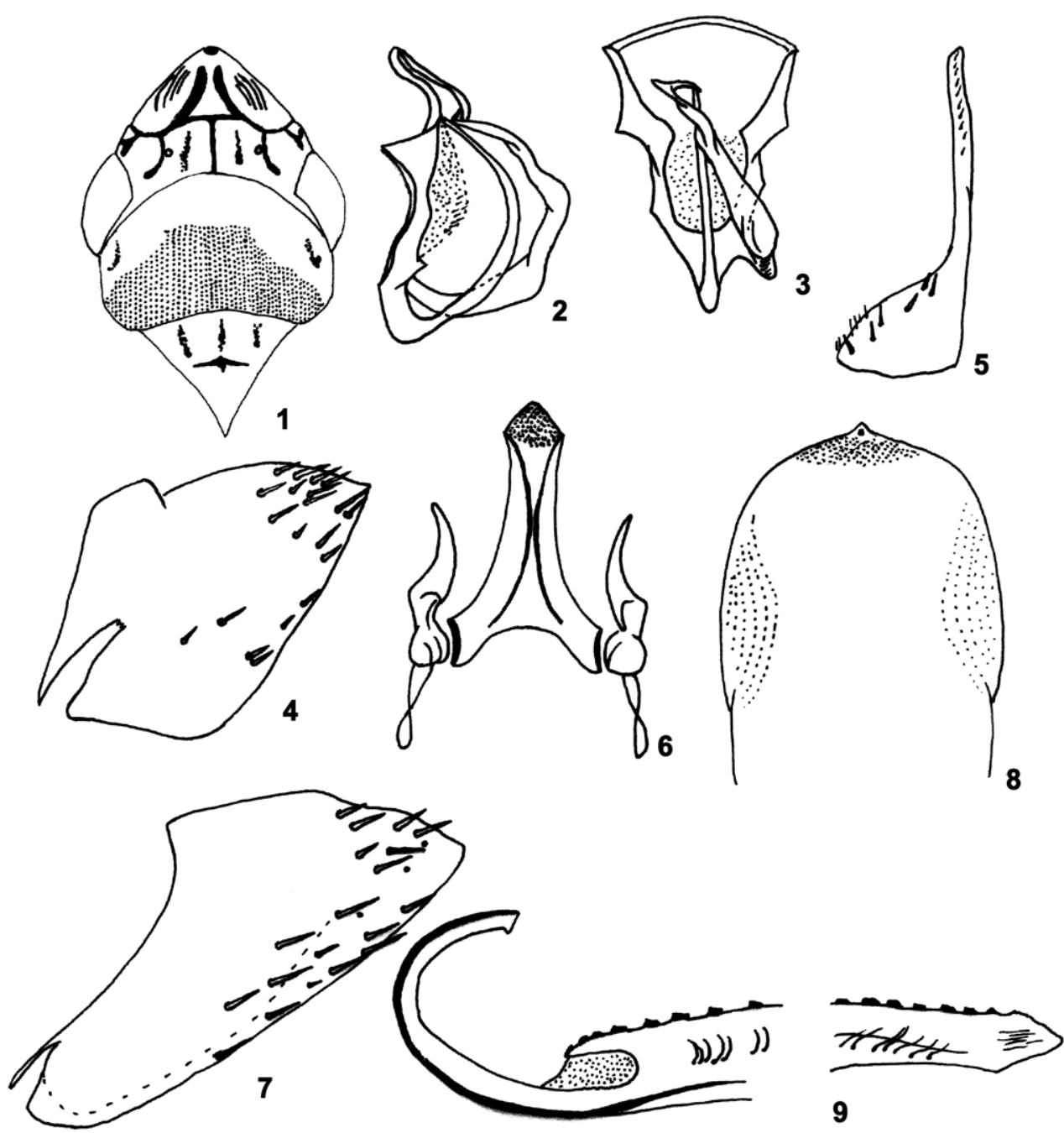

9

Figuras 1-9. Fingeriana dubia sp. nov. (1-6) Holótipo: (1) cabeça, pronoto e escutelo, em vista dorsal; (2) edeago, vista lateral; (3) edeago, vista posterior; (4) pigóforo do macho, em vista lateral; (5) placa subgenital, em vista ventral; (6) estilos e conetivo, em vista dorsal; (7-9) Alótipo: (7) pigóforo, em vista lateral; (8) VII esternito, em vista ventral; (9) segunda valvula, base e ápice.

do que longo, sem estrias. Asas anteriores quatro vezes mais longas do que sua maior largura, com três células anteapicais fechadas, a base da célula anteapical interna mais proximal que as demais; com quatro células apicais, a base da terceira mais proximal do que a da quarta; sem veias extranumerárias e plexo de veias. Pernas posteriores, com fórmula setal femural 2.1.1.; tarsômeros basais de comprimento maior do que a soma dos dois distais; superfície plantar com duas fileiras de microcerdas.

Base do abdômen com um par de apódemas robustas e truncadas apicalmente.

Pigóforo mais longo do que largo, obtuso apicalmente, sem processos, com macrocerdas distribuídas desde o terço basal. Placa subgenital triangular, bipartida, tão longa quanto o pigóforo, afilada desde o terço basal, com uma fileira de macrocerdas lateralmente, apenas no terço basal. Estilos curtos, delgados, ápice afilado e voltado para fora. Conetivo longo, robusto e em "Y". Edeago simétrico, delgado, fortemente curvado para trás; braços longos; com um processo assimétrico, originando-se no braço direito do edeago, curvado para trás e ápice em gancho.

Fêmea. Semelhante ao macho, levemente maior. Pigóforo obtuso, mais longo do que a largura basal, macrocerdas no terço apical. VII esternito longo, levemente mais curto do que o pigóforo, margem apical com uma projeção triangular medianamente. Segunda válvula com ápice triangular e com dentículos; margem dorsal com dentes primários quadrangulares na porção mediana.

Comentários. Fingeriana gen. nov. está relacionado com Nielsonia Young, 1977, Kapateira Young,1977, Macunolla Young, 1977 e Gillonella Nielson \& Godoy, 1995, porém, apresenta a cabeça mais triangular e produzida; edeago simétrico e com 
um processo assimétrico originando-se na base do braço direito do edeago. Sendo que os gêneros citados acima apresentam o edeago assimétrico, exceto Kapateira, que não apresenta processo em sua base.

No aspecto geral, Fingeriana gen. nov. é similar às espécies de Nielsonia, exceto pela coloração da cabeça e dos aspectos citados acimas. Outro fator importante é a distribuição das espécies de Nielsonia, que estão restritas a região Norte da América do Sul e apresentam além da característica do edeago assimétrico, duas manchas escuras, mais ou menos, arredondadas no pronoto.

\section{Fingeriana dubia sp. nov. Figs $1-9$}

Holótipo. Macho. "Limeira - S[ão] P[aulo]; BR[asil] - 1996; Yamamoto leg." (DZUP) [Coleção Entomológica Pe. Jesus Santiago Moure do Departamento de Zoologia da Universidade Federal do Paraná].

Alótipo. Mesmos dados do holótipo. (DZUP).

Parátipos. 4 우, “Lavras - M[inas] G[erais] - Brasil; Campus UFLA [Universidade Federal de Lavras]; Café Catuaí V/01" (DZUP) 1 ox e 9 우 "Bebedouro - SP; Brasil VIII-97; W. Peiffer col.; Cultura Poncã" (DZUP) $7 \sigma^{\pi} \sigma^{x}$ e 31 우 우 "Bebedouro SP; Brasil IV-97; W. Peiffer col." (DZUP), (1 ơ e 1 \%) depositado no Museu Nacional do Rio de Janeiro - MNRJ. 1 o e 3 우우, "Limeira - SP; Brasil - 1996; Yamamoto leg." 1 ơ e 1 , "Araraquara - SP; Brasil XII-1996; FUNDECITRUS-AM" $1 \sigma^{*} \mathrm{e}$ 4 우, "Ribeirão do Pinhal - PR; 01-99; Meneguim AM; CaféCampo"(DZUP).

Medidas. Holótipo/Alótipo. Comprimento total: 6,04/ 6,72; Comprimento mediano da cabeça: 0,80/0,92; Distância transocular: 1,56/1,72; Distância interocular: 0,84/1,12; Comprimento mediano do pronoto:0,80/0,92; Distância entre os úmeros: 1,36/1,52; Comprimento das asas anteriores: 4,68/5,16; Largura das asas anteriores: 1,08/1,24.

Coloração. Cabeça, dorsalmente, verde-amarelada com uma mancha preta na forma de um " $\mathrm{Pi}^{\mathrm{i}}(\pi)$ invertido, situada da linha média para o ápice, sendo os ramos convergentes e digitiformes; ápice com uma mancha arredondada, preta. Três faixas pretas longitudinais, duas localizadas lateral aos ocelos e uma ao longo da sutura coronal. Impressões musculares enegrescidas. Pronoto verde-amarelado, com o terço posteior mais escuro. Escutelo verde-amarelado com três faixas pretas unindose a linha preta localizada sobre o sulco mediano. Asas anteriores verdes, apicalmente translúcidas e escurecidas.

Comentários. Fingeriana dubia sp. nov. difere das espécies dos gêneros relacionados a Fingeriana gen. nov. pelo aspecto geral da coloração, principalmente, pela mancha preta localizada na região anterior, semelhante a um " $\mathrm{Pi}$ " $(\pi)$ invertido, além das estruturas da genitália do macho e pelo processo basal do edeago assimétrico.

Etimologia. Alusivo a dúvida que foi criada para enquadrá-la em gêneros relacionados.

\section{AGRADECIMENTOS}

Agradeço ao Conselho Nacional de Desenvolvimento Científico e Tecnológico (CNPq), pelo auxílio concedido referente ao processo 475565/01-0 e pela Bolsa de Produtividade em Pesquisa.

\section{REFERÊNCIAS BIBLIOGRÁFICAS}

CAVICHIOLI, R.R. 1996. Albiniana, gen.n. e revalidação de Alocha Melichar, 1926 (Homoptera, Cicadellidae, Cicadellinae). Revista Brasileira de Zoologia, Curitiba, 13 (3): 677-683. . 1998. Clypelliana mejdalanii gen.n. e sp.n. (Homoptera, Cicadellidade, Cicadellinae) do Mato Grosso, Brasil. Revista Brasileira de Zoologia, Curitiba, 15 (3): 617-619. 2001a. Jeepiulus flavus gen.n and sp.n. (Hemiptera, Auchenorhyncha, Cicadellidae) from Mato Grosso and Rondônia, Brazil. Insecta Mundi, Gainesville, 14 (4): 221224.

. 2001b. Wolfniana gen.n. and redescription of Wolfniana limbatula (Osborn, 1926) comb. nov. (Hemiptera, Auchenorrhyncha, Cicadellidae). Insecta Mundi, Gainesville, 14 (4): 253-255.

Hamilton, K.G.A. 1981. Morphology and evolution of the rhynchotan head (Insecta: Hemiptera, Homoptera). The Canadian Entomologist, Ottawa, 113: 953-974.

- 1985. Review of Draeculacephala Ball (Homoptera, Auchenorrhyncha, Cicadellidae). Entomologische Abhandlungen und Berichte aus dem Staatlichen Museum fur Tierkunde, Dresden, 49 (5): 83-103.

Mejdalani, G.L. 1994. A new genus of Cicadellini from Brazil. Revista Brasileira de Biologia, Rio de Janeiro, 54 (2): 219222.

1998. Morfologia externa dos Cicadellinae (Homoptera, Cicadellidae): comparação entre Versigonalia ruficauda (Walker) (Cicadellini) e Tretogonia cribrata Melichar (Proconiini), com notas sobre outras espécies e análise da terminologia. Revista Brasileira de Zoologia, Curitiba, 15 (2): 451-544.

Nielson, M.W. 1965. A revision of the genus Cuerna (Homoptera, Cicadellidae). Technical Bulletin. United States Department of Agriculture, Washington, D.C., 1318: 1-48.

Nielson, M.W. \& C. Godoy. 1995. A new genus and new species of cicadelline Leafhoppers from Costa Rica. (Cicadellidae, Cicadellinae). Contributions on Entomology International, Gainesville, 29 (2):189-204.

OMAn, P.W. (1949): The Nearctic leafhoppers (Homoptera: Cicadellidae). A generic classification and check list. Memoirs of Entomological Society of Washhington, D.C., 3: 1-253.

TakiYa, D.M., G.L. Mejdalani \& M. Felix. 2001. A new genus and species of Cicadellini (Hemiptera Cicadellidae Cicadellinae) from Southeastern Brazil. Tropical Zoology, Firenze, 14 (1):175-183

Young, D.A. 1968. Taxonomic study of the Cicadellinae (Homoptera: Cicadellidae). Part 1, Proconiini. United States Naional Museum Bulletin, Washington, D.C., 261: 1-287. 1977. Taxonomic study of the Cicadellinae (Homoptera: Cicadellidae). Part 2, New World Cicadellini and the genus Cicadella. Technical Bulletin North Carolina Agriccultural Experiment Station, North Carolina, 239: $1-1135$.

ZANOL, K.M.R. 1988. Morfologia de Neophlepsius gracilis (Osborn, 1923)(Homptera, Cicadellidae, Deltocephalinae). Revista Brasileira de Entomologia, São Paulo, 32 (1):69-93.

Recebido em 05.XII.2002; aceito em 13.V.2003.

Revista Brasileira de Zoologia 20 (2): 247-249, junho 2003 